\title{
Correction to: Correlation between indoor air pollution and adult respiratory health in Zunyi City in Southwest China: situation in two different seasons
}

Shixu Li, Jie Xu, Zhigang Jiang, Ya Luo, Yu Yang and Jie Yu*

\section{Correction to: BMC Public Health (2019) 19:723}

https://doi.org/10.1186/s12889-019-7063-z

It has been highlighted that the original article [1] contained some errors in the Result section of the Abstract. The incorrect and correct statement is shown in the Correction article.

Incorrect

The odds ratio (OR) for asthma-like symptoms and asthma in adults using coal stove for cooking or warming, relative to non-users, was $1.73(95 \% \mathrm{CI}, 1.11-2.69)$ in winter vs. 1.30 (95\% CI, $0.79-2.14)$ in summer.

\section{Correct}

The odds ratio (OR) for asthma-like symptoms and asthma in adults using coal stove for cooking or warming, relative to non-users, was 1.84 (95\% CI, $1.20-2.81)$ in winter vs. $2.32(95 \% \mathrm{CI}, 1.35-3.98)$ in summer.

Received: 8 July 2019 Accepted: 8 July 2019

Published online: 05 August 2019

\section{Reference}

1. Li S, et al. Correlation between indoor air pollution and adult respiratory health in Zunyi City in Southwest China: situation in two different seasons. BMC Public Health. 2019;19:723. https://doi.org/10.1186/s12889-019-7063-z.

\footnotetext{
* Correspondence: Xujie360@hotmail.com

School of Public Health, Zunyi Medical University, Zunyi, Guizhou 563000,

People's Republic of China
} 